\title{
How sensitive is a neutrino factory to the angle $\theta_{13}$ ?
}

\author{
P. Huber, ${ }^{1,2,3, \text { f T. Schwetz, }}$, 周 and J. W. F. Valle ${ }^{1, \text { f }}$ \\ ${ }^{1}$ Instituto de Física Corpuscular - C.S.I.C., \\ Universitat de València Edificio Institutos, Aptdo. 22085, E-46071 València, Spain \\ ${ }^{2}$ Theoretische Physik, Physik Department, Technische Universität München, \\ James-Franck-Strasse, D-85748 Garching, Germany \\ ${ }^{3}$ Max-Planck-Institut für Physik, Postfach 401212, D-80805 München, Germany
}

(Dated: 19.11.2001)

\begin{abstract}
We consider the impact of non-standard interactions of neutrinos (NSI) on the determination of neutrino mixing parameters at a neutrino factory using $\stackrel{(-)}{\nu} \rightarrow \stackrel{(-)}{\nu})$ "golden channels" for the measurement of $\theta_{13}$. We show how a small residual NSI leads to a drastic loss in sensitivity in $\theta_{13}$, of up to two orders of magnitude. This can be somewhat overcome if two baselines are combined.
\end{abstract}

The long-standing solar and atmospheric neutrino anomalies [1] now give compelling evidence for new physics in the lepton sector. The data are well described in the simplest 3-neutrino oscillation framework [2] defined by two mass-squared differences $\Delta m_{\text {sol }}^{2}$ and $\Delta m_{\text {atm }}^{2}$, three angles $\theta_{12}, \theta_{23}, \theta_{13}$ and one relevant $\mathrm{CP}$-violating phase $\delta$. The parameters $\Delta m_{\text {atm }}^{2}$ and $\theta_{23}$ are determined by atmospheric experiments, while $\Delta m_{\text {sol }}^{2}$ and $\theta_{12}$ are determined by solar experiments. For $\theta_{13}$ there is only an upper bound $\sin ^{2} 2 \theta_{13} \lesssim 0.1$ at $90 \%$ CL derived from reactor experiments [3]. The phase $\delta$ is completely unknown.

A new generation of long-baseline neutrino oscillation experiments using a neutrino beam from the decay of muons in a storage ring is discussed [4]. Such so-called neutrino factories should give a precise determination of $\theta_{13}$, possibly down to few $\times 10^{-4}$, exploring also the important issue of leptonic $\mathrm{CP}$ violation [5].

In a large class of models neutrino masses are accompanied by non-standard interactions of neutrinos (NSI). These can be non-universal (NU) or flavour-changing (FCI), and may arise also in the absence of neutrino mass [6]. Such non-standard interactions of neutrinos affect their propagation in matter, with implications for solar $17,8,9,10$, atmospheric 11, 12], and astrophysical neutrino sources 13. The possibility to use a neutrino factory for probing NSI with the earth matter, which neutrinos have to cross necessarily in a longbaseline experiment, has been previously examined in the appearance channels $\stackrel{(-)}{\nu_{e}} \rightarrow\left(\stackrel{-}{\nu_{\tau}} \sqrt{14}\right.$ and $\stackrel{(-)}{\nu}) \rightarrow \stackrel{(-)}{\nu}, 114,15$.

In this letter we will consider the impact of NSI on the determination of neutrino mixing parameters assuming the standard setup of a neutrino factory. Therefore, we will focus on the $\stackrel{(-)}{\nu}) \rightarrow(\stackrel{(-)}{\nu})$ channels, i. e. the "golden channels" for the measurement of $\theta_{13}$. In contrast to refs. 114, 15] we need only to assume a muon detector, which seems more realistic than a tau detector. We treat NSI and neutrino oscillations in a common framework to investigate correlations between the corresponding parameters. We show that the presence of a small FCI in the $e-\tau$ channel, safely within current limits, leads to a drastic loss in sensitivity in $\theta_{13}$, up to two orders of magnitude. This follows from a strong correlation between these parameters. We show how this effect can be diminished to some extent if two baselines are combined.

In the presence of NSI the evolution of neutrinos passing through matter is modified by a nonstandard potential due to the coherent forward scattering amplitude of NSI-processes $\nu_{\alpha}+f \rightarrow$ $\nu_{\beta}+f$, where $f$ is a fermion in the medium. For simplicity we will consider only NSI with downquarks: $f=d$. Then the potential induced by NSI can be written as $V_{\mathrm{NSI}}=\sqrt{2} G_{F} N_{d} \epsilon_{\alpha \beta}$, where $N_{d}$ is the number density of down-quarks along the neutrino path. The small numbers $\epsilon_{\alpha \beta}$ describe the NSI in units of the Fermi-constant $G_{F}$. The off-diagonal elements $\epsilon_{\alpha \beta}$ with $\alpha \neq \beta$ correspond to FCI, whereas the differences in the diagonal elements $\epsilon_{\alpha \alpha}$ lead to NU interactions. For simplicity we take all $\epsilon_{\alpha \beta}$ real. Present data on various lepton flavour violating processes can be used to set upper bounds on FCI 10, 16, 17 leading to $\left|\epsilon_{e \mu}\right| \lesssim 7 \times 10^{-5},\left|\epsilon_{\mu \tau}\right| \lesssim 5 \times 10^{-2},\left|\epsilon_{e \tau}\right| \lesssim 7 \times 10^{-2}$. Much weaker bounds of order 0.1 apply for the NU coefficients.

In this letter we show how claimed sensitivities on $\theta_{13}$ get destroyed if a small NSI component exists. For this it suffices to consider a simplified scenario in which we neglect the solar mass-squared difference. If we parameterize the lepton mixing matrix $U_{23} U_{13} U_{12}$, this implies also that the angle $\theta_{12}$ and the phase $\delta$ disappear. Further, we restrict ourselves to the elements $\epsilon_{e \tau}$ and $\epsilon_{\tau \tau}$ (the case $\epsilon_{\mu \tau}$ and $\epsilon_{\tau \tau}$ is considered in Ref. 115]), where present 
constraints are rather weak. Then the evolution of flavour neutrinos is governed by the Hamiltonian

$$
\begin{aligned}
H_{\nu}= & U_{23} U_{13} \operatorname{diag}(0,0, \Delta) U_{13}^{\dagger} U_{23}^{\dagger} \\
& +V\left(\begin{array}{ccc}
1 & 0 & r \epsilon_{e \tau} \\
0 & 0 & 0 \\
r \epsilon_{e \tau} & 0 & r \epsilon_{\tau \tau}
\end{array}\right) .
\end{aligned}
$$

Here we have defined $\Delta:=\Delta m_{\mathrm{atm}}^{2} / 2 E_{\nu}$ and $V=\sqrt{2} G_{F} N_{e}$ is the matter potential due to the Standard Model charged current interaction [8], where $N_{e}$ is the electron number density and $r:=$ $V_{\mathrm{NSI}} / V=N_{d} / N_{e}$ with $r \approx 3$ in earth matter. In the Hamiltonian (11) a sign change of $\Delta$ is equivalent to a sign change of $V$, which interchanges the evolution of neutrinos and anti-neutrinos. In the following we show that even in this simple scenario the strong correlation between $s_{13}$ and $\epsilon_{e \tau}$ will lead to serious complications for the measurement of $\theta_{13}$ at a neutrino factory.

To get an intuitive understanding of the numerical results it is useful to derive first an analytic expression for the appearance probability. To this aim we assume a constant matter potential $V$ and, since $s_{13}$ must be small, consider all terms containing $s_{13}, \epsilon_{e \tau}$ and $\epsilon_{\tau \tau}$ as a small perturbation of the Hamiltonian and calculate eigenvalues and eigenvectors of eq. 1 up to first order in these small quantities. The resulting appearance probability is second order and has the form

$$
P_{\nu_{e} \rightarrow \nu_{\mu}} \approx A s_{13}^{2}+B s_{13} \epsilon_{e \tau}+C \epsilon_{e \tau}^{2} .
$$

where the coefficients $A, B$ and $C$ are of the same order and depend on the neutrino energy, on the baseline $L$ and on the sign of $V$ (neutrinos or antineutrinos) in a nontrivial way. For example,

$$
A=4 s_{23}^{2}\left(\frac{\Delta}{\Delta-V}\right)^{2} \sin ^{2} \frac{(\Delta-V) L}{2}
$$

with similar expressions for $B$ and $C$. These analytic expressions for the appearance probabilities are in agreement with numerical calculations within a few $\%$ in the relevant parameter range. We note that the NU coefficient $\epsilon_{\tau \tau}$ does not show up in leading order in the appearance probability [14. Therefore, we will neglect it in our numerical studies and concentrate only on FCI. We consider both signs for $\epsilon_{e \tau}$, this sign however becomes irrelevant as $s_{13}$ approaches zero.

Eq. 2 implies that the lines of constant event rates in the $s_{13}-\epsilon_{e \tau}$ plane are ellipses, whose coefficients are obtained by fixing a baseline and folding $A, B$ and $C$ with flux and detection cross section. In fig. 1 we draw these ellipses for neutrinos

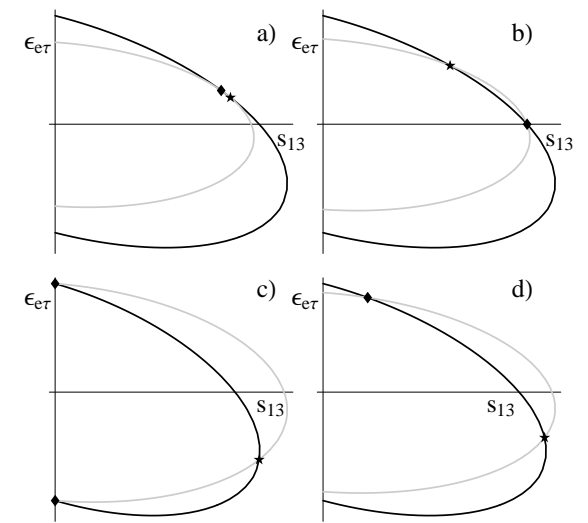

Figure 1: Lines of constant event rates in $s_{13}-\epsilon_{e \tau}$ (black for neutrinos and grey for anti-neutrinos) at $L=3000 \mathrm{~km}$.

and anti-neutrinos through different start points marked with a star. In general the two ellipses will cross not only at the start point. All points where the ellipses cross will lead to identical total event rates and are therefore a source of possible confusion. Depending on the choice of initial values four typical cases can be distinguished. Case (a) has two solutions witch are very close and away from both axes. In this case both NSI and oscillations can be established. Case (b) has a second solution on the $\epsilon_{e \tau}=0$ axes. Therefore one can only establish the existence of oscillations. Case (c) has additional solutions on the $s_{13}=0$ axes, which means that only NSI can be established but not oscillation. Case (d) has solutions which are close to both axes. If the count rate is not high enough the allowed region will touch both axes, leading to complete confusion between NSI and oscillation. In the following we explore this through a detailed numerical example and show that all these four cases can actually arise for realistic oscillation and NSI parameter choices.

In our numerical calculations we assume a neutrino factory with an energy of $50 \mathrm{GeV}$ for the stored muons and $2 \times 10^{20}$ useful muon decays of each polarity. We consider a magnetized iron detector with perfect charge identification of the muons and a mass of $10 \mathrm{kt}$. The muon detection threshold is set to $4 \mathrm{GeV}$, the energy resolution of the detector is approximated by a Gaussian resolution function with $\Delta E_{\nu} / E_{\nu}=10 \%$ and we use 20 bins in muon energy. The appearance probability is obtained by numerically solving the neutrino evolution equation with the Hamiltonian (11), using a realistic earth matter density profile. Then this probability is folded with neutrino flux, cross sec- 
tion and energy resolution function to obtain the event rates in the detector, following [18].

We consider only the $\nu_{e} \rightarrow \nu_{\mu}$ appearance channel since it contains most of the information. Our "observables" are the event rates of this channel $n_{\nu}^{i}\left(n_{\bar{\nu}}^{i}\right)$ for (anti-)neutrinos in each energy bin $i$. We fix the atmospheric oscillation parameters at $\Delta m_{\text {atm }}^{2}=3 \times 10^{-3} \mathrm{eV}^{2}$ and $\sin ^{2} 2 \theta_{23}=1$. Thus, at a given baseline the event rates depend on $S_{13}:=$ $\sin ^{2} 2 \theta_{13}$ and $\epsilon_{e \tau}$. In order to evaluate the potential of a neutrino factory to probe these parameters we proceed as follows. For any given $\left(S_{13}^{0}, \epsilon_{e \tau}^{0}\right)$ choice we calculate the event rates $n_{x}^{i}\left(S_{13}^{0}, \epsilon_{e \tau}^{0}\right)$ with $x=\nu, \bar{\nu}$ and construct a $\chi^{2}\left(S_{13}, \epsilon_{e \tau} ; S_{13}^{0}, \epsilon_{e \tau}^{0}\right)$ appropriate for a Poisson distribution. Using this we derive allowed regions in the $S_{13}-\epsilon_{e \tau}$ plane as usual. For all $S_{13}$ and $\epsilon_{e \tau}$ values inside these confidence regions the data can be fitted at the given CL (in our case 99\%) if the true values are $S_{13}^{0}$ and $\epsilon_{e \tau}^{0}$. Thus, these regions indicate the capability of a neutrino factory to probe $S_{13}$ and $\epsilon_{e \tau}$.

In fig. 2 we show such fits for four characteristic test points $\left(S_{13}^{0}, \epsilon_{e \tau}^{0}\right)$ at a baseline of $3000 \mathrm{~km}$ in correspondence with the cases considered in fig. 1 . The two grey lines passing through each of these test points are the lines of constant total rates for neutrinos and anti-neutrinos. From the fact that the confidence regions follow closely the lines of constant rates we learn that most information is contained in the total rates; the spectral information is not very important. We have verified that our results are, indeed, rather insensitive to variations of the number of energy bins and of the energy resolution. Moreover we find that one must take into account simultaneously both neutrino and anti-neutrino rates (this requires to run the neutrino factory in both polarities). This is easy to understand since the allowed regions extend as long as the lines of constant neutrino and antineutrino rates remain close to each other, i. e. both rates are similar to the ones at the test point. In regions where the lines are far from each other the neutrino or the anti-neutrino rates are very different from the one in the test point, and hence it is not possible to fit the data generated by the parameters $\left(S_{13}^{0}, \epsilon_{e \tau}^{0}\right)$ in those regions.

Let us consider in more detail the fits for the four different test points in fig. 2. For case (a) with large $S_{13}^{0}$ and $\epsilon_{e \tau}^{0}$ values one would need both oscillations and FCI. In case (b) $\left(S_{13}^{0}=10^{-2}, \epsilon_{e \tau}^{0}=\right.$ $\left.10^{-2}\right)$ the allowed region extends to the $\epsilon_{e \tau}=0$ axis. This means that the data in a neutrino factory generated by these parameters require oscillations. In contrast, for case (c) $\left(S_{13}^{0}=10^{-3}, \epsilon_{e \tau}^{0}=\right.$

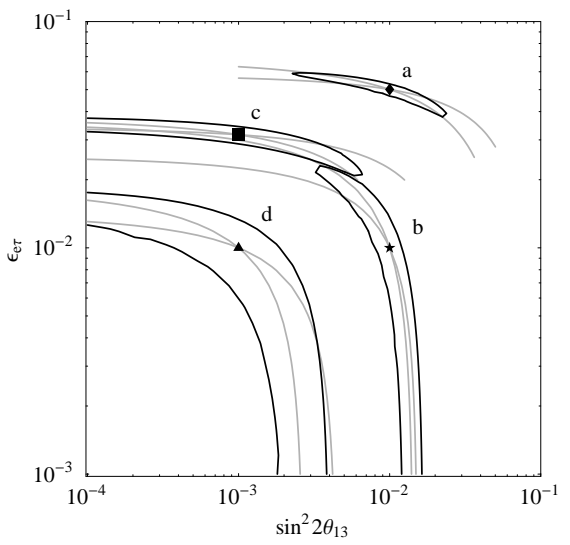

Figure 2: 99\% CL allowed regions (black lines) in the $\sin ^{2} 2 \theta_{13}-\epsilon_{e \tau}$ plane for different input values, as indicated by the points, at a baseline of $3000 \mathrm{~km}$. Lines of constant event rates are displayed in grey.

$3.2 \times 10^{-2}$ ) the allowed region extends only to the $S_{13}=0$ axis, indicating that the data in a neutrino factory generated by these parameters require the presence of FCI. Finally, in case (d) $\left(S_{13}^{0}=10^{-3}, \epsilon_{e \tau}^{0}=10^{-2}\right)$ there is complete confusion between oscillations and FCI, because the allowed region extends to both axes $\epsilon_{e \tau}=0$ and $S_{13}=0$. This means that the data in a neutrino factory generated by these parameters can be fitted either with pure oscillations $\left(\epsilon_{e \tau}=0\right)$ or pure FCI $\left(S_{13}=0\right)$ with both signs of $\epsilon_{e \tau}$. We can define a sensitivity to $S_{13}$ by requiring that the allowed region for a given point in the $S_{13}-\epsilon_{e \tau}$ plane does not touch the $S_{13}=0$ axis. By scanning the $S_{13}-\epsilon_{e \tau}$ plane we obtain the following sensitivity limits for three baselines:

\begin{tabular}{c|ccc} 
baseline & 700 & 3000 & 7000 \\
\hline w/o NSI & $2 \times 10^{-4}$ & $3 \times 10^{-4}$ & $5 \times 10^{-4}$ \\
w NSI & $1 \times 10^{-1}$ & $7 \times 10^{-2}$ & $5 \times 10^{-3}$
\end{tabular}

Comparing with the sensitivity limits without NSI [- $[$ ] we can see that the sensitivity loss is dramatic, especially for shorter baselines.

The geometry of the muon storage rings currently discussed offers the striking possibility to illuminate two detectors at different baselines with neutrinos from one neutrino factory. In fig. 3 we show the $99 \%$ CL region from a fit to data generated from the test point $S_{13}^{0}=10^{-3}$ and $\epsilon_{e \tau}^{0}=10^{-3}$ at $700 \mathrm{~km}$, at $7000 \mathrm{~km}$ and from a combination of both. In this particular case, for each of the two baselines taken alone there is complete confusion between $S_{13}$ and $\epsilon_{e \tau}$. However, combining the two baselines we obtain a much smaller 


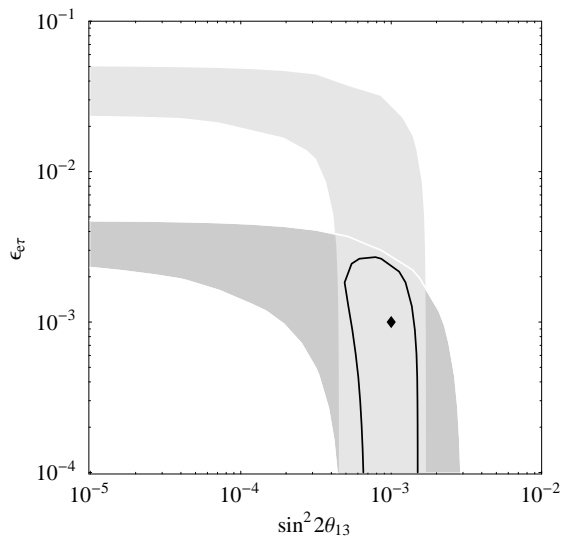

Figure 3: $\sin ^{2} 2 \theta_{13}-\epsilon_{e \tau}$ sensitivities when combining two baselines at $700 \mathrm{~km}$, at $7000 \mathrm{~km}$, see text.

allowed region indicated by the solid line, which is roughly given by the intersection of the individual regions. Here the data can be fitted with $\epsilon_{e \tau}=0$, with improved sensitivity for $S_{13}$. For the combination of two baselines the analysis is performed as before, but using the sum of the $\chi^{2}$-functions for the individual baselines. We have evaluated neutrino factory sensitivities (defined as above) for all possible combinations of the three baselines 700 $\mathrm{km}, 3000 \mathrm{~km}$ and $7000 \mathrm{~km}$.

\begin{tabular}{c|ccc} 
baseline & $700 \& 3000$ & $700 \& 7000$ & $3000 \& 7000$ \\
\hline w/o NSI & $2 \times 10^{-4}$ & $3 \times 10^{-4}$ & $3 \times 10^{-4}$ \\
w NSI & $2 \times 10^{-3}$ & $2 \times 10^{-3}$ & $2 \times 10^{-3}$
\end{tabular}

The sensitivities for $S_{13}$ are still considerably worse (up to one order of magnitude) than those for the pure oscillation case (no NSI). Notice that no substantial improvement is expected by changing the energy of the stored muons since neutrinos carry a relatively wide spectrum already for our nominal $50 \mathrm{GeV}$ choice.

We have shown within a simplified scenario that the presence of even a small NSI component, predicted in most models of neutrino mass, can deteriorate the sensitivity of a neutrino factory for $\theta_{13}$ by orders of magnitude. This problem can partly be resolved by combining two baselines. Additional effects of NSI in neutrino source and detection [19], and $\mathrm{CP}$ violating phases in NSI coefficients, as well as in the mixing matrix will lead to even more serious difficulties [20].

This work was supported by the European Commission grants HPRN-CT-2000-00148 and HPMT2000-00124, by the ESF Neutrino Astrophysics Network and by Spanish MCyT grant PB98-0693. We thank discussions with H. Nunokawa whose work 14 stimulated our interest.

* Electronic address: phuber@ph.tum.de

$\dagger$ Electronic address: schwetz@ific.uv.es

¥ Electronic address: valle@ific.uv.es

[1] Talks by Y. Totsuka and K. Heeger at http://neutrinhouches.in2p3.fr/.

[2] M.C. Gonzalez-Garcia, et al., Phys. Rev. D 63 (2001) 033005.

[3] M. Apollonio, et al., CHOOZ Coll., Phys. Lett. B 466 (1999) 415.

[4] C. Quigg, Nucl. Instrum. Meth. A 451 (2000) 1; C. Albright, et al., hep-ex/0008064; B. Autin, et al., CERN-SPSC-98-30; S. Geer, Phys. Rev. D 57 (1998) 6989 [Erratum-ibid. D 59 (1998) 039903]; M. Freund, P. Huber and M. Lindner, Nucl. Phys. B 615 (2001) 331; V. Barger, et al., Phys. Rev. D 62 (2000) 073002.

[5] K. Dick, et al., Nucl. Phys. B 562 (1999) 29.

[6] J. Schechter and J. W. F. Valle, Phys. Rev. D 22, 2227 (1980); R. N. Mohapatra and J. W. F. Valle, Phys. Rev. D 34 (1986) 1642. L. J. Hall, V. A. Kostelecky and S. Raby, Nucl. Phys. B 267 (1986) 415; R. Adhikari, A. Sil and A. Raychaudhuri, hep-ph/0105119.

[7] J.W.F. Valle, Phys. Lett. B 199 (1987) 432.

[8] L. Wolfenstein, Phys. Rev. D 17, 2369 (1978); S.P. Mikheev and A.Yu. Smirnov, Sov. J. Nucl. Phys. 42, 913 (1985).

[9] M. M. Guzzo, et al., hep-ph/9908308; V. Barger, R. J. N. Phillips and K. Whisnant, Phys. Rev. D44, 1629 (1991); S. Bergmann, Nucl. Phys. B515, 363 (1998); E. Ma and P. Roy, Phys. Rev. Lett. 80, 4637 (1998).

[10] S. Bergmann et al, Phys. Rev. D 62 (2000) 073001.

[11] M.C. Gonzalez-Garcia, et al., Phys. Rev. Lett. 82 (1999) 3202; N. Fornengo, M.C. Gonzalez-Garcia and J.W.F. Valle, JHEP 0007 (2000) 006; P. Lipari and M. Lusignoli, Phys. Rev. D 60 (1999) 013003; G.L. Fogli, et al., Phys. Rev. D 60 (1999) 053006 .

[12] N. Fornengo, et al., hep-ph/0108043.

[13] H. Nunokawa, et al., Phys. Rev. D 54 (1996) 4356; D. Grasso, H. Nunokawa and J.W.F. Valle, Phys. Rev. Lett. 81 (1998) 2412.

[14] A.M. Gago, et al., Phys. Rev. D 64 (2001) 073003.

[15] P. Huber and J.W.F. Valle, hep-ph/0108193, Phys. Lett. B, in press.

[16] S. Bergmann and Y. Grossman, Phys. Rev. D 59 (1999) 093005.

[17] S. Bergmann, Y. Grossman and D.M. Pierce, Phys. Rev. D 61 (2000) 053005.

[18] M. Freund, P. Huber and M. Lindner, Nucl. Phys. B 585 (2000) 105.

[19] M. C. Gonzalez-Garcia, et al., Phys. Rev. D 64 (2001) 096006.

[20] P. Huber, et al., in preparation. 